\title{
The $p \bar{p}$ mass threshold structure in $\psi(3686)$ radiative decay revisited
}

\author{
J. Haidenbauer ${ }^{1}$ and Ulf-G. Meißner ${ }^{1,2}$ \\ ${ }^{1}$ Institute for Advanced Simulation, Institut für Kernphysik, \\ and Jülich Center for Hadron Physics, \\ Forschungszentrum Jülich, D-52425 Jülich, Germany \\ ${ }^{2}$ Helmholtz Institut für Strahlen- und Kernphysik and Bethe Center for Theoretical Physics, \\ Universität Bonn, D-53115 Bonn, Germany
}

\begin{abstract}
The near-threshold behavior of the $p \bar{p}$ invariant mass spectrum from the $\psi(3686) \rightarrow \gamma p \bar{p}$ decay reported recently by the BESIII Collaboration is analyzed. The enhancement in the $p \bar{p}$ invariant mass spectrum near threshold is nicely reproduced by the $p \bar{p}$ final-state interaction based on the isospin averaged ${ }^{1} S_{0}$ partial-wave amplitude as predicted by the Jülich nucleon-antinucleon model. Contributions from the $f_{2}(1910)$ or $f_{2}(1950)$ mesons, as promoted in earlier works, are not needed.

PACS numbers: 12.39.Pn; 13.25.Gv; 13.75.Cs; 25.43.+t
\end{abstract}

Recently, the BESIII Collaboration presented data with improved statistics on the $p \bar{p}$ invariant mass spectrum for the reaction $J / \psi \rightarrow \gamma p \bar{p}$, but also a first highstatistics measurement of the $\psi(3686) \rightarrow \gamma p \bar{p}$ decay [1]. The new $J / \psi \rightarrow \gamma p \bar{p}$ measurement confirmed the spectacular near-threshold enhancement in the $p \bar{p}$ invariant mass, found in an earlier experiment by the BES Collaboration [2], which has been seen as evidence for a $p \bar{p}$ bound state or baryonium [3 $[\underline{6}$ ], for exotic glueball states [7, 8$]$, but also simply as manifestation of the final-state interaction (FSI) between the outgoing proton and antiproton 9 15]. A significant near-threshold enhancement of the $p \bar{p}$ invariant mass was seen also in the $\psi(3686)$ decay, although less pronounced than in the $J / \psi$ case.

The BESIII Collaboration themselves interpreted their $J / \psi$ decay data in terms of the $p \bar{p}$ FSI proposed by us [9], but folded with a Breit-Wigner type resonance at around $1835 \mathrm{MeV}$ presuming that the structure at the $p \bar{p}$ threshold might be related to the $X(1835)$ resonance that had been observed in the reaction $J / \psi \rightarrow \gamma \pi^{+} \pi^{-} \eta^{\prime}$ [16, 17], see also the comments in Ref. [10]. This object is called $X(p \bar{p})$ in Ref. [17]. For the description of the $\psi(3686)$ decay the same $X(p \bar{p})$ amplitude is used but sizeable additional contributions from the $f_{2}(1910)$ resonance had to be invoked. Contributions of a tensor meson, but in this case of the $f_{2}(1950)$, were also advocated in the work of the CLEO collaboration [18], which had published data on the reaction $\psi(3686) \rightarrow \gamma p \bar{p}$ decay prior to BESIII, though with lower statistics. In both cases an isoscalar meson with a larger mass $\left(f_{0}(2100)\right.$ and $f_{2}(2150)$, respectively) has been added to explain the $p \bar{p}$ spectrum at higher invariant masses.

In this report we take a closer look at those $\psi(3686) \rightarrow \gamma p \bar{p}$ data from the BESIII Collaboration. Specifically, we provide an alternative interpretation of the near-threshold enhancement solely in terms of the $p \bar{p}$ FSI, i.e. without resorting to any resonance contributions like the $f_{2}(1910)$ or $f_{2}(1950)$ (or the $X(1835)$ ), based on the very same $N \bar{N}$ interaction used by us previously in the explanation of the enhancement in the $J / \psi$ decay [9].

Conservation laws for parity, charge-conjugation and total angular momentum severely restrict the partial waves in the $p \bar{p}$ system [9] for such decay processes. Specifically, the partial-wave analysis for the $J / \psi$ decay performed in [1] suggests that the near-threshold enhancement is dominantly in the $J^{P C}=0^{-+}$state, which means that the $p \bar{p}$ system should be in the ${ }^{1} S_{0}$ partial wave (we use here the standard nomenclature ${ }^{(2 S+1)} L_{J}$ where $S$ is the total spin and $L$ the orbital angular momentum). However, since the decay of the $J / \psi$ and $\psi(3686)$ to the $\gamma p \bar{p}$ system involves electromagnetic processes, isospin is not conserved so that, in principle, any combination of the isospin $I=0$ and $I=1$ components is allowed. Indeed, while the $p \bar{p}$ invariant mass for $J / \psi$ decay can be understood in terms of the FSI generated by the isospin $I=1$ component of the $N \bar{N}$ amplitude in the ${ }^{1} S_{0}$ state alone - at least in our work $[9]$ - the $I=1$ and $I=0$ channels can occur with different weights in case of the $\psi(3686)$ decay.

The $\psi(3686) \rightarrow \gamma p \bar{p}$ decay rate is given by $[9]$

$$
\begin{aligned}
& d \Gamma=\frac{|A|^{2}}{2^{9} \pi^{5} m_{\psi}^{2}} \lambda^{1 / 2}\left(m_{\psi}^{2}, M^{2}, m_{\gamma}^{2}\right) \\
& \times \lambda^{1 / 2}\left(M^{2}, m_{p}^{2}, m_{p}^{2}\right) d M d \Omega_{p} d \Omega_{\gamma},
\end{aligned}
$$

where the Källén function $\lambda$ is defined by $\lambda(x, y, z)=$ $\left((x-y-z)^{2}-4 y z\right) / 4 x, M \equiv M_{p \bar{p}}$ is the invariant mass of the $p \bar{p}$ system, $\Omega_{p}$ is the proton angle in that system, while $\Omega_{\gamma}$ is the $\gamma$ angle in the $\psi(3686)$ rest frame. After averaging over the spin states and integrating over the angles, the differential decay rate is

$$
\frac{d \Gamma}{d M}=\frac{\left(m_{\psi}^{2}-M^{2}\right) \sqrt{M^{2}-4 m_{p}^{2}}}{2^{7} \pi^{3} m_{\psi}^{3}}|A|^{2} .
$$

The quantity $A$ in Eqs. (10) and (2) stands for the total $\psi(3686) \rightarrow \gamma p \bar{p}$ reaction amplitude and is dimensionless.

We assume again the validity of the Watson-Migdal [19, 20] approach for the treatment of the FSI effect. It suggests that the reaction amplitude for a production and/or decay reaction that is of short-ranged nature can be factorized in terms of an elementary (basically constant) production amplitude $A_{0}$ and the $p \bar{p}$ scattering 


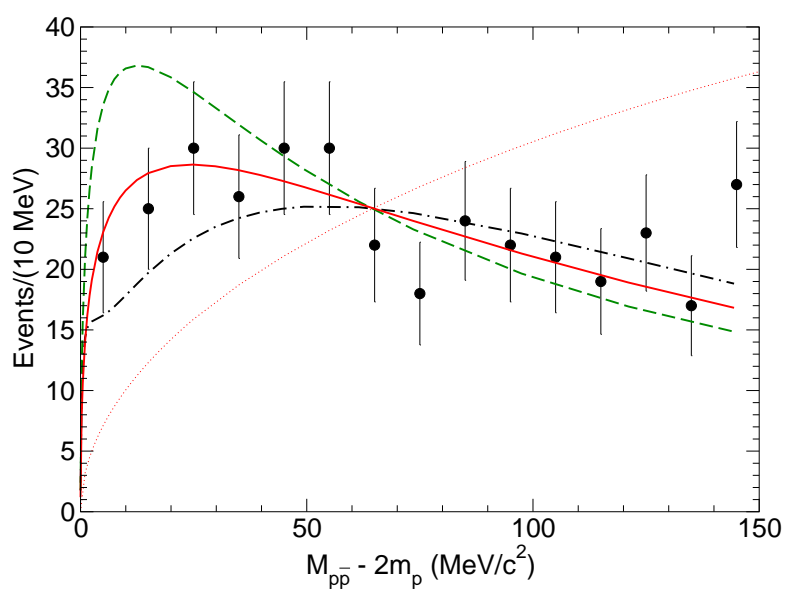

FIG. 1: The $p \bar{p}$ mass spectrum from the decay $\psi(3686) \rightarrow \gamma p \bar{p}$. The circles show experimental results of the BES Collaboration [1]. The solid line is a calculation using the $p \bar{p}$ scattering amplitude squared $\left(\left|\left(T_{I=1}+T_{I=0}\right) / 2\right|^{2}\right)$ predicted by the $N \bar{N}$ model A(OBE) 21, 22] for the ${ }^{1} S_{0}$ partial wave, appropriately normalized to the data, cf. Eq. (3). The dashed (dash-dotted) curve are corresponding results using the $I=1(I=0)$ amplitudes alone while the dotted line is the spectrum obtained from Eq. (2) by assuming a constant reaction amplitude $A$. Those curves were normalized so that they all coincide at $M_{p \bar{p}}-2 m_{n} \approx 60 \mathrm{MeV}$.

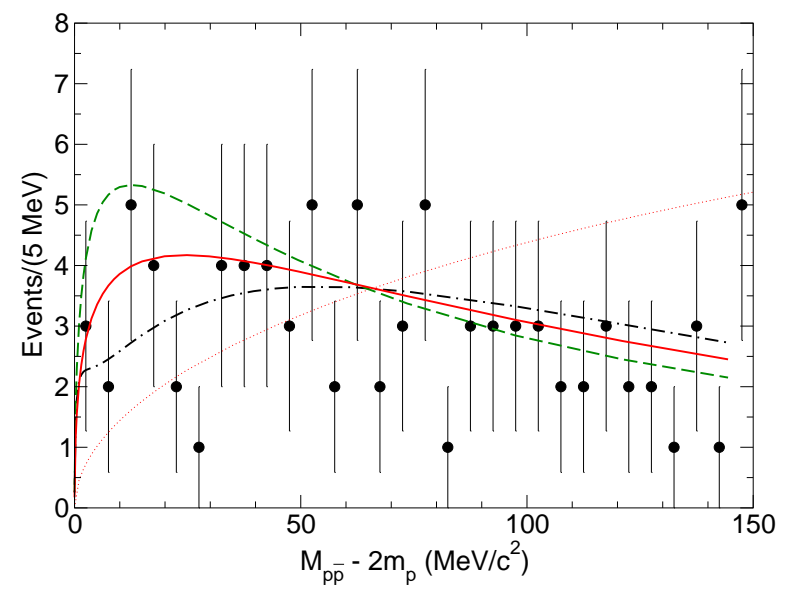

FIG. 2: The $p \bar{p}$ mass spectrum from the decay $\psi(3686) \rightarrow \gamma p \bar{p}$. The circles show experimental results of the CLEO Collaboration [18]. For notation of curves, see Fig. 1]

amplitude $T$ of the particles in the final state so that

$$
A\left(M_{p \bar{p}}\right) \approx A_{0} \cdot N \cdot T\left(M_{p \bar{p}}\right),
$$

where $N$ is an arbitrary normalization factor, see e.g. Ref. 9] for further details. As in our investigation of the $J / \psi$ decay we employ the amplitudes predicted by the $N \bar{N}$ model A(OBE) published in Refs. [21, 22], and we assume that the FSI effects in the $\psi(3686)$ decay are likewise dominated by the ${ }^{1} S_{0}$ partial wave.

Our results are presented in Fig. 1 together with the data from Ref. [1]. As expected from the curves pre- sented in Fig. 3 of Ref. [1], the $N \bar{N}$ amplitude in the $I=1$ channel, which successfully describes the rather strong enhancement detected in the reaction $J / \psi \rightarrow \gamma p \bar{p}$ [9], overestimates the energy dependence seen in the $\psi(3686)$ case, cf. the dashed curve. On the other hand, the result based on the isospin averaged amplitude, $\left(T_{I=1}+T_{I=0}\right) / 2 \equiv T_{p \bar{p}}$, shown in Fig. 1 by the solid line, agrees rather nicely with the energy dependence found in the experiment. With an appropriately chosen normalization, cf. Eq. (3), the data are well reproduced from the $p \bar{p}$ threshold up to excess energies of about 150 $\mathrm{MeV}$. In particular, the $\chi^{2}$ is 8.7 for the 15 data points shown in Fig. 1 while it is 22.2 , i.e. more than twice as large, for the pure $I=1$ amplitude. We also include the result based on FSI effects due to the $p \bar{p}$ amplitude in the $I=0$ channel alone (dash-dotted curve) and we indicate the pure phase-space behaviour by the dotted curve. The latter is obtained by using a constant amplitude $A$ in Eq. (2). The $\chi^{2}$ value for the pure $I=0$ amplitude is 11.9. The one for the phase-space curve amounts to 60 which is a clear indication that the measured invariant mass spectrum does not exhibit a phase-space behaviour near threshold. All those curves are normalized to the solid curve at $M_{p \bar{p}}-2 m_{p} \approx 60 \mathrm{MeV}$ in order to facilitate a comparison of the differences in the energy dependence.

Based on those findings we do not see any need here to invoke further more substantial contributions coming from any $f_{2}(1910)$ or $f_{2}(1950)$ mesons, say, as done in Refs. [1, 18], in order to explain the data.

In Fig. 2 our results are compared with the data obtained by the CLEO Collaboration [18]. Given the poorer statistics it is difficult to infer the actual energy dependence of the $p \bar{p}$ invariant mass. Still, the solid curve corresponding to the FSI effects based on $T_{p \bar{p}}=\left(T_{I=1}+T_{I=0}\right) / 2$, again appropriately normalized, comes closest to the trend exhibited by the data. But in terms of the $\chi^{2}$ the differences are marginal. All results including FSI effects yield $\chi^{2} /$ data $\approx 20 / 30$ while the pure phase space amounts to $30 / 30$.

Finally, in Fig. 3 the results for BESIII are displayed again, however this time in terms of the modulus squared of the amplitude $A$. Here the curves correspond directly to the (appropriately normalized) scattering amplitude squared $\left(|T|^{2}\right)$ predicted by the $N \bar{N}$ model A(OBE) [21, 22 for the ${ }^{1} S_{0}$ partial wave. The symbols indicate the experimental values of $|A|^{2}$, obtained from the BESIII data [1] via dividing the latter by the kinematical factors according to Eq. (2).

In summary, we have analyzed the near-threshold behavior of the $p \bar{p}$ invariant mass spectrum from the $\psi(3686) \rightarrow \gamma p \bar{p}$ decay reported recently by the BESIII Collaboration within the Watson-Migdal approach. Although in this reaction there is definitely an enhancement in the near-threshold region as compared to the phasespace behavior, it is much less pronounced than what was found for the corresponding reaction $J / \psi \rightarrow \gamma p \bar{p}$. The enhancement is nicely reproduced by the $p \bar{p}$ final-state interaction based on the isospin averaged ${ }^{1} S_{0}$ partial-wave 


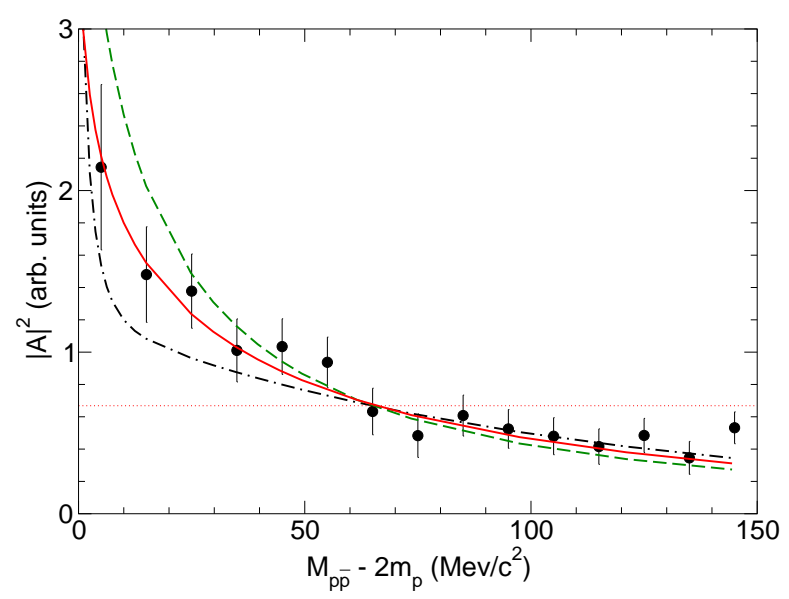

FIG. 3: Invariant $\psi(3686) \rightarrow \gamma p \bar{p}$ amplitude $|A|^{2}$ as a function of the $p \bar{p}$ mass. The circles symbolize the experimental values of $|A|^{2}$ extracted from the BES data [1] via Eq. (2). The curves are the appropriately normalized scattering amplitude squared, $|T|^{2}$, predicted by the $N \bar{N}$ model A(OBE) [21, 22] for the ${ }^{1} S_{0}$ partial wave. For notation of curves, see Fig. 1 .

amplitude as given by the Jülich $N \bar{N}$ model. In par- ticular, any more substantial contributions from tensor mesons like $f_{2}(1910)$ or $f_{2}(1950)$, as advocated in earlier works [1, 18], are not required.

Note that we have used here the same $N \bar{N}$ amplitudes as in our study of the $J / \psi$ decay [9]. In the $J / \psi$ case the FSI provided by the $I=1$ component alone led to an agreement with the measured near-threshold $p \bar{p}$ invariant mass spectrum. Clearly, the mechanisms for the decay of the $J / \psi$ and $\psi(3686)$ mesons into $\gamma p \bar{p}$ should be different so that different admixtures of the two isospin components in the final $p \bar{p}$ state have to be expected. Only dedicated microscopic calculations, which hopefully will be performed in the future, can allow to shed light on the details of the reaction mechanisms.

\section{Acknowledgments}

This work is supported in part by the DFG and the NSFC through funds provided to the Sino-German CRC 110 "Symmetries and the Emergence of Structure in QCD", and by the European Community-Research Infrastructure Integrating Activity "Study of Strongly Interacting Matter" (acronym HadronPhysics3).
[1] M. Ablikim et al. [BESIII Collaboration], Phys. Rev. Lett. 108, 112003 (2012).

[2] J.Z. Bai et al., Phys. Rev. Lett. 91, 022001 (2003).

[3] A. Datta and P.J. O'Donnell, Phys. Lett. B 567, 273 (2003).

[4] G. J. Ding and M. L. Yan, Phys. Rev. C 72, 015208 (2005).

[5] M. Suzuki, J. Phys. G 34, 283 (2007).

[6] J.-P. Dedonder, B. Loiseau, B. El-Bennich and S. Wycech, Phys. Rev. C 80, 045207 (2009).

[7] C. K. Chua, W. S. Hou and S. Y. Tsai, Phys. Lett. B 544, 139 (2002).

[8] J.L. Rosner, Phys. Rev. D 68, 014004 (2003).

[9] A. Sibirtsev, J. Haidenbauer, S. Krewald, U.-G. Meißner and A. W. Thomas, Phys. Rev. D 71, 054010 (2005).

[10] J. Haidenbauer, U.-G. Meißner and A. Sibirtsev, Phys. Rev. D 74, 017501 (2006).

[11] B. Kerbikov, A. Stavinsky, and V. Fedotov, Phys. Rev. C 69, 055205 (2004).

[12] D.V. Bugg, Phys. Lett. B 598, 8 (2004).
[13] B.S. Zou and H.C. Chiang, Phys. Rev. D 69, 034004 (2004).

[14] B. Loiseau and S. Wycech, Phys. Rev. C 72, 011001 (2005).

[15] D. R. Entem and F. Fernández, Phys. Rev. D 75, 014004 (2007).

[16] M. Ablikim et al. [BES Collaboration], Phys. Rev. Lett. 95, 262001 (2005).

[17] M. Ablikim et al. [BESIII Collaboration], Phys. Rev. Lett. 106, 072002 (2011).

[18] J. P. Alexander et al. [CLEO Collaboration], Phys. Rev. D 82, 092002 (2010).

[19] K.M. Watson, Phys. Rev. 88, 1163 (1952).

[20] A.B. Migdal, JETP 1, 2 (1955).

[21] T. Hippchen, J. Haidenbauer, K. Holinde, V. Mull, Phys. Rev. C 44, 1323 (1991).

[22] V. Mull, J. Haidenbauer, T. Hippchen, K. Holinde, Phys. Rev. C 44, 1337 (1991). 